\title{
THE INFLUENCE OF SELECTING A SPECIFIC SAMPLE OF HAZARDOUS EVENTS ON THE EVALUATION OF THE TRAIN DRIVER'S RELIABILITY
}

\author{
Dipl.-Ing. Tobias Lindner \\ Technische Universität Braunschweig, Germany \\ Institute of Railway Systems Engineering and Traffic Safety
}

\begin{abstract}
The safety of railway operation is influenced by the possibility of the occurrence of technical and human errors. For evaluating the human influence on safety, different approaches exist. One possibility is an analysis of accident descriptions from hazardous events. Within this method, the user can identify the underlying influential factors by analyzing the separate events. As a basis for the evaluation, all events appearing on a large network could be considered, the sample is very large. However, this must not lead to significant results, inevitably. Instead, it has to be questioned if a large railway network features different properties which may influence the occurrence of a human error. This could be infrastructural as well as operational parameters, for example. A change within one of the parameters could lead to a change of the error occurrence or rather a modified range of a specific performance shaping factor. In this case, the evaluation of a large network containing different properties would lead to insignificant results. Instead, it can make sense to choose a selection of the events that should be considered for the evaluation or rather to filter the events on specific parameters.
\end{abstract}

The present article discusses the shift of contributing factors if different operational and infrastructural properties are assumed as a basis for the evaluation.

\section{Introduction}

In the development of innovations to enhance train drivers' workplaces and operational procedures, it is important to evaluate their role in hazards appearing during railway operation. One possible approach is to conduct an analysis of accident descriptions from hazardous events which occurred within a specific 
railway network in the past. For each failure scenario, e.g. the passing of a signal at danger (SPAD), data of the concrete events should allow the user to identify the underlying influential factors. After that, the probability that a specific factor leads to the hazardous situation can be calculated.

Experience in this field has shown a wide range of operational and infrastructural constraints that may influence not only the quantity of events but also the set of performance shaping factors contributing to human errors. For getting significant results in any case, one possibility is to define a sample railway line with specific operational and infrastructural properties. In the further evaluation, only hazardous events whose operational and infrastructural background fits with the sample railway line are considered

Evaluations have shown that it has to be discussed to which extent the probability of the occurrence of a hazardous event will change if operational constraints are modified, i.e. the properties of the sample railway line that were taken as a reference. For example, it can be expected that the numbers of errors based on perception will decrease if the allowed speed limit on a given railway line is lowered and all other factors remain constant.

The variety of the operational constraints considered in the following chapters is derived from the typical operational modes and configurations of the German railway system. Different properties will be evaluated with respect to SPADs as one mostly occurring example of failure scenario. The evaluation takes the alteration potential and corresponding influence on the probability of this kind of hazardous event into account.

In the paper, the question about getting insignificant results if doing the evaluation without filtering the events for specific constraints will be discussed in a qualitative scope as well as if it is possible to transfer results that are based on a sample railway line to an infrastructure or rather railway system featuring different properties. Based on long-year experience, examples of different infrastructural and operational parameters will be identified and evaluated. It will also be discussed, if a change of the parameters could lead to a modification of the range of a performance shaping factor's occurrence. This can accompany a change of the probability of hazardous events. The qualitative evaluation may serve as a basis for a subsequent quantitative analysis, not only for the network of the German railways but also for transferring the complexity of problems to perform a similar research for railway systems for other countries. It will be evaluated concerning SPADs, that were identified by Kim et al. (2006) as the mostly occurring failure scenario in practice.

\section{Infrastructural and operational parameters}

The following infrastructural and operational parameters present some examples with potential of influencing the appearance of human errors. The different 
evaluated parameters and their type of being influenced are summarized in Table 1. Some of the parameters are purely based on infrastructure or operation, other parameters are primarily based on one of those two types and secondary on the corresponding other type.

Table 1: Summary of different exemplified parameters

\begin{tabular}{|c|c|} 
Parameter & Type \\
\hline Maximum speed and visibility & infrastructural \\
\hline Station infrastructure & infrastructural \\
\hline Signalling system & infrastructural (or/and operational) \\
\hline Operational mode & operational \\
\hline Changes of timetable & operational (or/and infrastructural) \\
\hline Changes of track allocation & operational (or/and infrastructural) \\
\hline
\end{tabular}

Maximum speed and visibility of the railway line

Explained by Kim et al. (2006), a SPAD is based on an inadequate track observance, in many cases. The speed of a train plays an important role, because this influences the time frame that is available for observing the signals located beside the track significantly, as a parameter of the visibility. For one specific, single signal, the probability of an error can get decreased, if a train drives with less speed. Thus, a slower train (e.g. freight train) passes a signal at danger with a lower probability, compared with a faster passenger train. This statement is only valid for a specific signal of a specific line that is used by both kinds of trains. It is not possible to transfer it to other lines, because in normal case, the visibility properties vary between each different line.

If the maximum speed of a train is not determined by the specific train's properties, especially in passenger operation a train normally drives with the maximum line speed. In this case, the maximum speed of operation is based on the track alignment. Because of centrifugal force specifications it is normally determined by the curve radiuses, the corresponding formulas are explained by Matthews (2007), for example. A curved alignment can also influence the visibility of a railway line. A dead straight railway line that can be operated with high speed provides an easy and broad visibility, whereas curved railway lines with lower maximum speed allow a larger time frame but less visibility to use it. Thus, a line operated with less speed does not necessarily lead to a decreasing probability of human errors concerning visibility than a fast line, in any case.

An optimal significant transfer of human error probabilities is only possible if speed and visibility difference compensate each other. Apart from other visibility influencing parameters as vegetation for example, a transfer of the results can be 
made if both infrastructures are used by trains driving with maximum speed determined by track alignment or rather with a consistent fractional amount of it.

As a conclusion, a broad interdependency between speed and visibility can be seen. It is qualitatively summarized by Figure 1 .

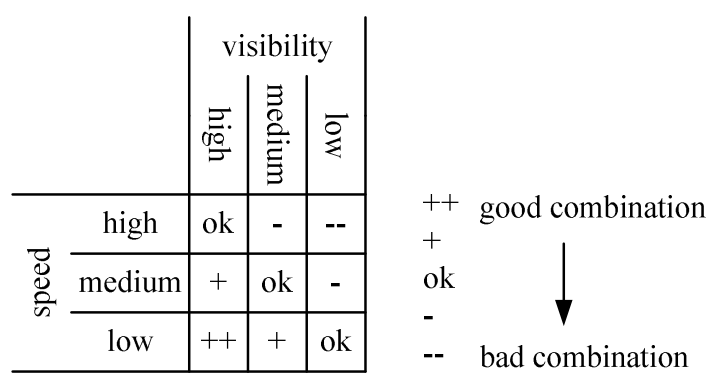

Figure 1: Correlation between speed and line visibility

\section{Station infrastructure}

The station design or rather the clearness of station infrastructure can also influence the appearance of human errors. If a station only contains one exit signal for each travelling direction, the single signal does not provide potential for being mistaken for a signal of a neighboring track. In contrast, especially larger station areas may lead to signal mistakes. The probability of appearance will not only increase by the complexity of station infrastructure with its number of signals, but also by the signal positions. Especially parallel located exit signals may lead to mistakes. An even worse case is existent, if the line between a stopping position in a station and the exit signal consist of a curve. A corresponding example is presented by Figure 2 . 


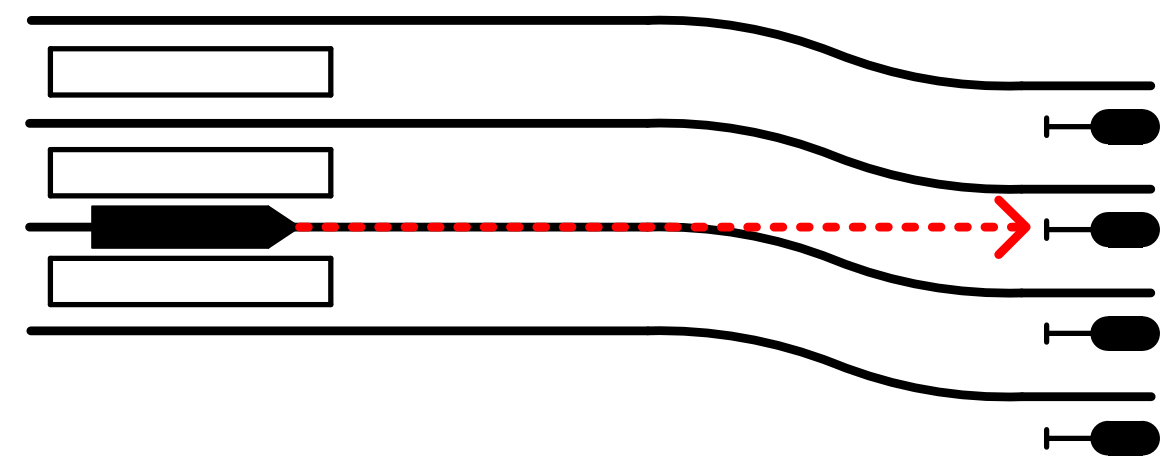

Figure 2: Situation with the potential of mistaking an exit signal of a station

Mistaking a signal can especially appear, if the exit signal of a neighboring track shows a "clear" signal aspect ("green"); the correct signal remains showing the "stop" aspect ("red"). In this case, the train driver may consider the signal of the neighboring track as valid and accelerates the train. If not recognizing the mistake during the process of acceleration, it will lead to a SPAD. The interdependency between mistaking a signal and track alignment also shows the connection to speed as an influencing parameter and their connection. Speed will always play a very important and higher-ranking role, because the time frame for recognizing a hazardous situation and the possibility of avoiding an accident is mainly influenced by the current speed of a train's movement.

\section{Signalling system}

Apart from different signal styles that may cause a different visibility, the potential of human error can be dependent on the existence of cabin (inside) or line (outside) signals. Using cabin signals, an observance of signals located outside could be redundant. That means the visibility of the outside signals and its error potential is not remaining any more. It can be assumed that the existence of a cabin signaling system increases the correct recognition of signals and their aspects.

If using a line signal system, the observance of the track and the signals can be done synchronized. Cabin signaling leads to a disruption of the line observance, because the driver has to shift the attention to the cabin instruments for getting the signal information. Thus, the positive aspects of the cabin signaling on safety may influence the observance of the track negatively.

\section{Operational mode}

One possible alternative to a signal-based distance spacing in case of normal operation, is the running of trains in a degraded procedure, as the on-sight mode. As described in Deutsche Bahn AG (2009), in the network of the German railways, on-sight mode is applied in case of signal failures or for doing shunting movements within a station area, for example. In the on-sight mode, the train is not protected by any signals. That means, the driver has to keep the speed very low, to guarantee 
a manual stop of the train if recognizing a barrier (for example another train or stabled wagons) on its track. In this case, the observance of the track plays a very important role. To guarantee an error-free execution of the on-sight mode, the maximum speed is restricted to $25 \mathrm{~km} / \mathrm{h}$. In general, the application of specific driving modes could require a modified task frame. This may also influence the occurrence of human errors. Some further general informations about running in on-sight mode at the network of German rather US railways are explained by Pachl (2002 and 2008).

\section{Changes of timetable and track allocation}

Changes of the timetable or track allocations as examples for unscheduled events can lead to a demand for train driver's unusual activities. Those activities can be connected with a higher stress level, inadequate knowledge how to handle the situation or rather a combination of both aspects, leading to an increasing human error potential.

A non-regarded change within the timetable at a stop in a station may lead to an untimely departure. A combination of station infrastructure and poor visibility etc. (see Figure 2) may lead to a mistaking of signals - especially if a neighboring signal switches into the "clear" aspect instead of the valid signal. Similar scenarios are possible if a train uses an alternative track unscheduled and its driver assumes to be in the usual track.

\section{Results}

Different examples have shown infrastructural as well as operational parameters that may influence the probability of human error appearance. The different parameters may affect the size of performance shaping factors. A strict division between the several properties is hardly possible, because the parameters may not only overlay but also influence each other. For example, the station infrastructure can be in context with the track visibility and the maximum speed, concerning the error occurrence.

\section{Transfer of results from one line to other trains of the same line}

Considering the mentioned parameters, a transfer of results from one line to other trains of the same line could be possible under specific circumstances. Of course, a the transfer within the same line does not lead to a change of infrastructure parameters. Thus, the visibility as well as the station infrastructure is constant. An ideal transfer of the results is possible, if the following preconditions are fulfilled.

- Trains running with same speed 
- Trains using same signaling system (this does not have to purely be determined by the infrastructure)

- Trains using same operational mode

- No (or same extent of) changes of timetable and track allocation

The more preconditions are not fulfilled, the less significance can be expected within the results.

Transfer of results from one line to trains of another line

Another possibility is to transfer the results from one line to trains of another line. For getting significant results, a maximum amount of preconditions mentioned above should also be fulfille. In addition, it has to be considered that apart from operational conditions, another line normally features different infrastructural properties. Thus, also the following should be considered for getting ideal significant results.

- $\quad$ Line are used with same speed

- Nearly same visibility conditions

Exceptional case: Both infrastructures are used by trains driving with maximum speed determined by track alignment or rather with a consistent fractional amount of it.

\section{Conclusion}

The paper shows that different parameters of infrastructure and operation may influence the human error occurrence. Under specific circumstances it could be possible to transfer the results based on a specific railway line with its operational constraints to another line or another kind of operation on the same line. In case of transfer, the difference between the parameters of reference and evaluating conditions should be considered in a first step. This allows an estimation of the transferability of the results by calculating its significance.

The exemplified presented parameters and the discussion of transferring results of a selected sample of hazardous events is not only a problem of specific national characteristics, but can be found in every railway network. Dependent on infrastructure and operation, thus for nation specific properties, parameters may vary within their quantity. A detailed quantitative analysis could make sense. 


\section{References}

Deutsche Bahn AG 2009, Richtlinie 408 - Züge fahren und Rangieren, valid from 13th December 2009, (DB Netz AG, Frankfurt)

Kim, J., Jung, W., Jang, S. and Wang, J. 2006, A case study for the selection of a Railway Human Reliability Analysis Method. Proceedings (International Railway Safety Conference 2006, Belfast)

Matthews, V. 2007, Bahnbau, Seventh Edition, (Vieweg and Teubner, Wiesbaden)

Pachl, J. 2002, Railway Operation and Control, First Edition, (VTD Rail Publishing, Mountlake Terrace)

Pachl, J. 2008, Systemtechnik des Schienenverkehrs, Fifth Edition, (Vieweg and Teubner, Wiesbaden) 